\title{
Especialización inteligente: una estrategia posible para Costa Rica
}

\author{
Smart specialization: a possible strategy for Costa Rica \\ Doi: https://doi.org/10.22458/rna.v1 1i2.3197
}

Dra. Marianela Cortés Muñoz, Phd

Universidad de Costa Rica, Costa Rica

marianela.cortes@ucr.ac.cr

https://orcid.org/0000-0002-9672-557X

Mag. Sharlín Sánchez Espinoza

Consejo Nacional de Rectores, Costa Rica

ssanchez@,conare.ac.cr

https://orcid.org/0000-0002-1755-7636
Licda. Cecilia Barrantes Ramírez

Universidad Estatal a Distancia, Costa Rica

cbarrantes@uned.ac.cr

https://orcid.org/0000-0001-9628-9141

Mag. Karla Halabi Guardia

Instituto Tecnológico de Costa Rica, Costa Rica

khalabi@itcr.ac.cr

https://orcid.org/0000-0002-4805-8354
Mag. Marjorie Marchena Bustos

Universidad Estatal a Distancia, Costa Rica mmarchena@uned.ac.cr

https://orcid.org/0000-0002-3495-9696

\section{RESUMEN}

La innovación es un factor estratégico del desarrollo económico, social y cultural del país que quiera alcanzar altos niveles de crecimiento y bienestar para su población.

El País Vasco hace más de 20 años viene gestando un modelo de innovación con actores del ecosistema mediante la Estrategia de Especialización Inteligente, que lo convierte en referente europeo y polo de innovación.

La puesta en marcha de estrategias "inteligentes", conlleva agendas integradas de transformación económica territorial, apoyo político e inversiones para el desarrollo a través de centros tecnológicos, de investigación, unidades $\mathrm{I}+\mathrm{D}$, clústeres estratégicos y universidades como actores clave generadores de conocimiento

\section{ABSTRACT}

Innovation is a strategic factor in the economic, social and cultural development of the country aiming at reaching high levels of growth and well-being for its population.

For over 20 years the Basque Country has been developing an innovation model with ecosystem stakeholders through the Smart Specialization Strategy, which makes it a European reference and an innovation hub.

The implementation of the "smart" strategies involves integrated agendas for territorial economic transformation, political support and investments for development through technological and research centers, R\&D units, strategic clusters and universities as key actors generating knowledge

\section{RÉSUMÉ}

L'innovation est un facteur stratégique de développement économique, social et culturel du pays qui vise à atteindre de hauts niveaux de croissance et de bien-être pour sa population. Depuis plus de 20 ans, le pays basque est engagé dans un modèle d'innovation avec des acteurs de l'écosystème au moyen de la Stratégie de la Spécialisation Intelligente, ce qui en fait une référence européenne et un pôle d'innovation.

La mise en marche de stratégies "intelligentes" implique des agendas intégrés de transformation économique, territoriale, appui politique et des investissements pour le développement à travers des centres technologiques, de recherche, des unités $\mathrm{I}+\mathrm{D}$, des clusters stratégiques et des universités comme acteurs clés pour générer des connaissances

\section{RESUMO}

A inovação é um fator estratégico do desenvolvimento econômico, social e cultural do país que queira atingir altos níveis de crescimento e bem-estar para a sua população.

O País Vasco, há mais de 20 anos, vem gestando um modelo de inovação com atores do ecossistema mediante a Estratégia de Especialização Inteligente, o que o torna um referente europeu e pólo de inovação.

A implementação de estratégias "inteligentes", conleva agendas integradas de transformação econômica territorial, apoio político e investimentos para o desenvolvimento através de centros tecnológicos, de investigação, unidades I + D, de clústeres estratégicos e universidades como atores chave geradoras de conhecimento

\section{PALABRAS CLAVE: \\ INNOVACIÓN, \\ ESTRATEGIA RIS3, \\ DESARROLLO \\ ECONÓMICO, \\ ESTRATEGIA \\ REGIONAL, POLOS DE \\ INNOVACIÓN.}

KEY WORDS:

INNOVATION, RIS3

STRATEGY, ECONOMIC

DEVELOPMENT,

REGIONAL STRATEGY

INNOVATION HUBS.
PALAVRAS CHAVE:

INOVAÇÃO,

ESTRATÉGIA RIS3,

DESENVOLVIMENTO

ECONÔMICO,

ESTRATÉGIA REGIONAL,

PÓLOS DE INOVAÇÃO.
MOTS CLÉS:

INNOVATION, STRATEGIE

RIS3, DEVELOPPEMENT

ECONOMIQUE,

STRATEGIE REGIONALE,

POLES D'INNOVATION. 


\section{INTRODUCCIÓN}

Nexo-Universidad Sector Socio Productivo es una Subcomisión interuniversitaria del Consejo Nacional de Rectores (CONARE) que promueve la articulación entre las cinco universidades públicas costarricenses y los sectores socioproductivos, esto mediante la vinculación externa y transferencia de conocimiento, con el fin de coadyuvar en el fortalecimiento de las capacidades del ecosistema costarricense de ciencia y tecnología para la innovación.

Durante más de 25 años esta subcomisión ha realizado diversas actividades con actores de la triple hélice y, más recientemente, ha trabajado en la promoción del concepto de quíntuple hélice con el fin de promover la divulgación y vinculación del quehacer de las universidades.

Conocer y estudiar modelos de innovación de zonas exitosas es una estrategia que ha sido de vital importancia para la subcomisión. Para esto, se planteó conocer la experiencia del País Vasco (Euskadi) en España, con el fin de analizar su modelo como polo de innovación de desarrollo económico, social y cultural y donde se visitaron su agencia de innovación "Innobasque", las universidades de la región, los centros de investigación y otras organizaciones relacionadas con el tema, de tal forma que su análisis y conclusiones puedan servir de base para promover un aumento en la innovación en Costa Rica.

\section{METODOLOGÍA}

El tema de Especialización Inteligente es introducido a la Subcomisión Nexo-Universidad Sector Socio Productivo del CONARE por parte de la Vicerrectoría de Investigación de la Universidad Estatal a Distancia (UNED) en el año 2017. Para ahondar en la temática, en ese mismo año, se realiza un taller que la Subcomisión denomina "Especialización Inteligente: Costa Rica hacia un polo de innovación" con el fin de generar mayor conocimiento en este campo entre los miembros del ecosistema país.

\section{Taller de especialización inteligente}

Se desarrolló un taller en San José, en las instalaciones de la UNED, con presentaciones de expertos sobre competitividad territorial y especialización inteligente, seguido del desarrollo de actividades con los participantes que permitieron plantear los entornos teóricos en razón de lo expuesto en las charlas y punto de partida para determinar preliminarmente el potencial de las regiones de nuestro país.

Los expertos que desarrollaron las conferencias fueron el Dr. Juan Carlos Montoya Castro de la Organización de los Estados Americanos (OEA), Especialista en Competitividad, Innovación y Tecnología, quien trató el tema “Competitividad Territorial en las Américas, Red Interamericana de Competitividad (RIAC)” y el Dr. José María Villate Blanco, Director Ejecutivo de la Agencia Vasca de la Innovación, quien presentó el tema "Experiencia de Euskadi-Basque Country en el desarrollo de la Estrategia de Especialización Inteligente". Los participantes del taller fueron investigadores de las universidades públicas, funcionarios del sector productivo, sector gobierno, representantes de ministerios, cámaras de empresarios y de la Promotora de Comercio Exterior (PROCOMER), entre otros.

Se organizaron mesas de trabajo y con la ayuda de una guía metodológica se desarrollaron los siguientes temas (Campos, 2017):

1. La ubicación en el mapa de cada región del país de aquellos puntos fuertes de actividad económica o áreas de oportunidad según los participantes en el taller, así como la forma de aprovechar los puntos destacados, además, se señaló la valoración sobre capacidades empresariales y científico-tecnológicas.

2. Aportes para una estrategia de especialización inteligente en Costa Rica en cinco ejes a saber: apoyo mediante políticas e inversiones en las prioridades, retos y necesidades clave del país o región para el desarrollo basado en el conocimiento; reconocimiento de los puntos fuertes, ventajas competitivas y 
potencial de excelencia de cada región; fomento de la innovación; estímulo de la inversión del sector privado y convocatoria e involucramiento de la sociedad civil.

3. Identificación de posibles sinergias para trabajo conjunto posterior al evento.

\section{Pasantía}

Con base en los resultados del taller de Especialización Inteligente realizado en la UNED, se elaboró una propuesta para la visita al País Vasco con el objetivo de analizar su modelo como polo de desarrollo económico, social y cultural que permitiera la identificación de elementos base para la elaboración de propuestas que promuevan el desarrollo de la innovación en el ecosistema nacional con apoyo de la Subcomisión Nexo Universidad Sector SocioProductivo. Previo a la visita, se realizó el análisis de documentos e informes relacionados con el tema la Estrategia de Especialización Inteligente que nutrió la elaboración de la propuesta presentada a las autoridades con el fin de llevar a cabo una observación in situ sobre las prácticas desarrolladas en el País Vasco en la temática.

Durante la pasantía, se siguió una metodología de observación participativa con entrevistas abiertas y visitas de campo a las diferentes organizaciones incluidas en el cronograma. Estas visitas y entrevistas generaron datos cualitativos sobre la metodología de Research and Innovation Smart (RIS3) en la cual se basa el éxito del País Vasco.

La agenda, compuesta de sesiones de trabajo con profesionales en diversos campos, la mayoría de ellos en el puesto de más alta jerarquía, permitió profundizar sobre la experiencia en la industria 4.0, cultura y políticas de innovación, innovación social, economía circular, investigación, internacionalización de la l+D+i vasca, generación de redes, plataformas de innovación y proyectos de cooperación con el programa Horizonte 2020.

Conocer la Agencia Vasca de la Innovación, Innobasque, asociación privada sin ánimo de lucro, que cuenta con líderes de los agentes científico-tecnológicos, del tejido empresarial y social y de la administración, fue el primer objetivo. Funciona como asesor del gobierno vasco y constituye una potente plataforma público-privada para la transformación socioeconómica de Euskadi, la cual aspira a ser el referente europeo en innovación. Además de visitar esta Agencia, se visitó y se entrevistó a autoridades de las universidades de Mondragón, País Vasco y Deusto y se visitó la Corporación Tecnalia, el Parque Tecnológico de Zamudio, el Euskampus y la IK4 Alianza de Investigación.

\section{RESULTADOS}

\section{Desarrollo de la Especialización inteligente}

En la era de la sociedad del conocimiento es cada vez más relevante que los países se enfoquen en el impulso de sus sistemas nacionales de ciencia, tecnología e innovación, ya que enfrentan entornos más complejos y se requiere encontrar soluciones innovadoras a las apremiantes necesidades de la sociedad frente a retos cada vez más exigentes, en un contexto donde los ciudadanos disponen de un acceso prácticamente ilimitado e inmediato a la información que promueven las innovaciones tecnológicas desarrolladas en especial las telecomunicaciones y el internet. La RIS3 ha sido propuesta por la Unión Europea y representó, en el año 2014, un cambio profundo en sus políticas de innovación. Se basa en 15 años de experiencia en el respaldo de las estrategias de innovación en las regiones y en el pensamiento económico de primera línea por parte de las principales instituciones internacionales, como el Banco Mundial, la Organización para la Cooperación y el Desarrollo Económico (OCDE) y el Fondo Monetario Internacional (FMI). Las zonas más avanzadas ya se han implicado en ejercicios estratégicos similares, como lo demuestra la iniciativa de Regiones por el Cambio Económico o el Monitor de Innovación Regional.

El Reglamento (UE) n 1301/2013 del Parlamento Europeo y del Consejo del 17 de diciembre 2013 es una base jurídica que define la estrategia de especialización inteligente, en la cual las estrategias de innovación nacionales o regionales definirán prioridades para crear ventajas competitivas mediante el desarrollo y la adecuación de los puntos fuertes de la investigación y la innovación a las necesidades empresariales, a fin de abordar las oportunidades emergentes y los avances del mercado de manera coherente y evitar, al mismo tiempo, la duplicación y fragmentación de los esfuerzos. Una estrategia de especialización inteligente puede incluirse en un marco político estratégico de investigación e innovación nacional o regional, o adoptar la forma de este. El desarrollo de las estrategias de 
especialización inteligente debe implicar la participación de las autoridades de gestión nacional o regional y de partes interesadas, como universidades y otras instituciones de enseñanza superior, la industria y los interlocutores sociales en el proceso de descubrimiento de emprendedores (Urkullu, 2014). La especialización inteligente consiste en aprovechar los puntos fuertes del ecosistema, las capacidades de investigación y las ventajas competitivas, así como el potencial de excelencia, propias de esa región.

Para conseguir este escenario, se deben alinear las políticas económicas e industriales, con las de innovación, ciencia y tecnología; es necesario concentrar los recursos disponibles para la generación y explotación del conocimiento en el contexto regional, al servicio de un número concreto de prioridades vinculadas a las fortalezas y ventajas competitivas, en materia de generación y explotación económica del conocimiento y provocar una orientación del tejido productivo hacia una senda de desarrollo económico.

\section{Taller de especialización inteligente (UNED)}

El taller tenía como objetivo principal conocer el expertise de los distintos participantes desde sus áreas de trabajo y sondear posibilidades de apoyo para el futuro además de dar a conocer la temática de especialización inteligente. Ya que esta estrategia promueve la identificación de las fortalezas de una determinada región para potenciar su desarrollo a través de la innovación y, a su vez, implica el reconocimiento de las áreas estratégicas con potencial nacional e internacional, y el nivel de desarrollo de capacidades empresariales y de las capacidades científico tecnológicas, se presenta a continuación, en la tabla 1, las percepciones de las personas que participaron en el taller sobre estos temas en relación con las regiones de Costa Rica.

Tabla 1.

Percepción del potencial en relación con las áreas de oportunidad o prioridades estratégicas, las capacidades científico-tecnológicas y las capacidades empresariales según regiones de Costa Rica

\begin{tabular}{|c|c|c|c|c|c|c|}
\hline \multicolumn{7}{|c|}{ Regiones } \\
\hline & Central & Brunca & Chorotega & Huetar Atlántica & $\begin{array}{r}\text { Pacífico } \\
\text { Central }\end{array}$ & Huetar Norte \\
\hline $\begin{array}{l}\text { Áreas de } \\
\text { oportunidad }\end{array}$ & $\begin{array}{l}\text { Servicios, } \\
\text { industrias, } \\
\text { sector } \\
\text { financiero, } \\
\text { gobierno, } \\
\text { sector } \\
\text { industrial } \\
\text { tecnológico, } \\
\text { turismo. }\end{array}$ & $\begin{array}{l}\text { Biodiversidad, } \\
\text { territorios } \\
\text { indígenas, } \\
\text { ecoturismo, } \\
\text { recursos } \\
\text { marino- } \\
\text { costero, } \\
\text { agroindustria. }\end{array}$ & $\begin{array}{l}\text { Turismo } \\
\text { sostenible, } \\
\text { ganadería, } \\
\text { energía, } \\
\text { folklor, } \\
\text { tradición y } \\
\text { artesanía, } \\
\text { zona azul. }\end{array}$ & $\begin{array}{l}\text { Logística, } \\
\text { agroindustria, } \\
\text { turismo, } \\
\text { conservación y } \\
\text { medioambiente. }\end{array}$ & $\begin{array}{l}\text { Turismo, } \\
\text { pesca, } \\
\text { logística y } \\
\text { desarrollo } \\
\text { portuario, } \\
\text { industria y } \\
\text { riqueza } \\
\text { marina. }\end{array}$ & $\begin{array}{l}\text { Ganadería y } \\
\text { producción } \\
\text { lechera, } \\
\text { agroindustria, } \\
\text { turismo, } \\
\text { recurso } \\
\text { hídrico, } \\
\text { turismo de } \\
\text { salud, } \\
\text { senvicios } \\
\text { tecnológicos, } \\
\text { turismo } \\
\text { ecológico, } \\
\text { energía, salud } \\
\text { y bienestar. }\end{array}$ \\
\hline $\begin{array}{l}\text { Capacidades } \\
\text { científico } \\
\text { tecnológicas }\end{array}$ & Alta & Media- baja & $\begin{array}{l}\text { Media- } \\
\text { Alta }\end{array}$ & Media & $\begin{array}{c}\text { Media- } \\
\text { baja }\end{array}$ & Media- alta \\
\hline $\begin{array}{l}\text { Capacidades } \\
\text { empresariales }\end{array}$ & Alta & Baja & $\begin{array}{l}\text { Media- } \\
\text { Alta }\end{array}$ & Media & $\begin{array}{c}\text { Media- } \\
\text { Alta }\end{array}$ & Media- alta \\
\hline
\end{tabular}

Fuente: elaboración propia. 
Este ejercicio es una primera aproximación en la identificación de esos parámetros. Se debe profundizar más en la determinación de las áreas de oportunidad que realmente puedan tener potencial en relación con las capacidades del sector empresarial y las capacidades relacionadas con los temas científicos y tecnológicos. En el país, se han dado algunas iniciativas locales o regionales en ese sentido, que en principio deberían permitir priorizar y concentrar los esfuerzos de la Quíntuple Hélice: gobierno, academia, sector empresarial y sociedad civil articulados en torno a la Sostenibilidad para promover la innovación y el desarrollo social y económico.

Entre las acciones regionales que se han desarrollado a nivel nacional encontramos las zonas económicas especiales como estrategias de desarrollo económico local donde se une la academia, el gobierno y el sector empresarial, con el fin de mejorar la competitividad de una región específica, impulsar el empleo de calidad en la zona lo que, a fin de cuentas, contribuye a impactar positivamente la calidad de vida de las personas (Instituto Tecnológico de Costa Rica, 2018).

\section{El País Vasco}

Es una comunidad autónoma española, situada en el extremo nororiental de la franja Cantábrica con una superficie de 7.234 $\mathrm{Km}^{2}$ y una población de 2,1 millones de habitantes. Está reconocida como nacionalidad histórica por su Estatuto de Autonomía y cuenta con tres provincias: Álava, cuya capital es Vitoria-Gasteiz; Guipúzcoa cuya capital es San Sebastián, Donostia; y Vizcaya, cuya capital es Bilbao.

El País Vasco, desde los años 80 y a raíz de la crisis económica vivida, decide apostar por la Estrategia de Especialización Inteligente (RIS3). Es a partir de esta decisión que se crean los centros tecnológicos, se dictan políticas de tecnología e innovación progresivas, se desarrolla una red de centros de investigación, unidades de I+D empresarial, clústeres con una perspectiva de fuerte articulación. La misión planteada fue hacer del País Vasco el referente europeo en innovación en el 2030.

\section{a. RIS3 y Comités de pilotaje}

La RIS3 pretende definir estrategias de $\mathrm{I}+\mathrm{D}+\mathrm{i}$ regionales que sean "inteligentes" en el sentido de concentrar sus recursos e inversiones en áreas donde existen claras sinergias con las capacidades productivas existentes y potenciales de la región.

Las estrategias de investigación e innovación nacionales y regionales para la especialización inteligente se ocupan de cinco asuntos importantes:

1. Se centran en el apoyo de la política y las inversiones en las prioridades, retos y necesidades clave del país o región para el desarrollo, basado en el conocimiento.

2. Aprovechan los puntos fuertes, ventajas competitivas y potencial de excelencia de cada país o región.

3. Respaldan la innovación tecnológica, así como la basada en la práctica, y aspiran a estimular la inversión del sector privado.

4. Involucran por completo a los participantes y fomentan la innovación y la experimentación.

5. Se basan en la evidencia e incluyen sistemas sólidos de supervisión y evaluación (Euskadi, 2014).

La especialización inteligente implica por ello, identificar las características y activos exclusivos de cada región, subrayar sus ventajas competitivas y reunir a los participantes en torno a una visión de futuro compartida.

El Consejo Vasco de Ciencia, Tecnología e Innovación, parte del plan de ciencia, tecnología e innovación, lo profundiza y lo mejora con el apoyo de 30 grupos de trabajo a los que llaman comités de pilotaje y que son integrados por los siguientes sectores: gobierno, empresas y centros de investigación. Estos grupos, se encargan de hacer la evaluación y la mejora de la metodología RIS3, de manera constante sobre los planes estratégicos definidos en cada sector.

Como se puede ver en la figura 1, en el País Vasco se han identificado tres ámbitos estratégicos: fabricación avanzada, energía y biociencias (esta última incluye el área de la salud), además de cuatro áreas de oportunidad: alimentación, planificación y regeneración urbana, ecosistemas y por último ocio, entretenimiento y cultura (Euskadi, 2014). 
Figura 1.

Ámbitos de oportunidad según capacidad empresarial y científico-tecno-

lógica en Euskadi

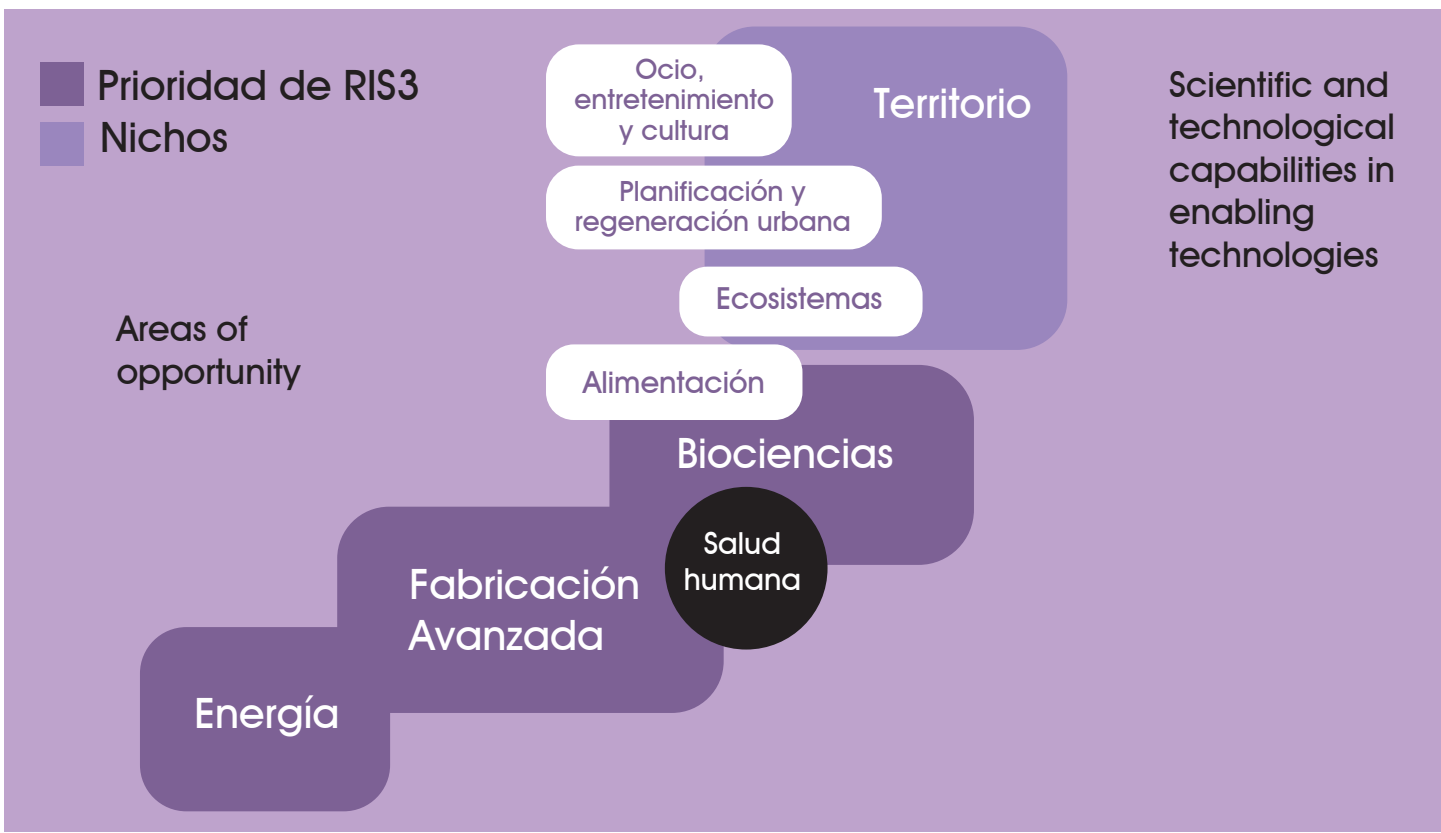

Potenciador exportador

Business sectors / Clusters

Demanda

Interna

Fuente: Euskadi, 2014.

\section{a. Papel de Innobasque}

Es indispensable resaltar los esfuerzos llevados a cabo desde Innobasque para reactivar una región europea como el País Vasco, donde se ha venido implementando la armonía franca y comprometida de la academia, las empresas, el gobierno local y otros agentes claves en pro de la innovación y el desarrollo económico, social, político y cultural.

Es una organización que impulsa, de forma prospectiva, todos aquellos aspectos relacionados con la ciencia, tecnología e innovación como parte de los activos indispensables de las empresas en su intencionalidad de incorporar el valor agregado, los nuevos retos y las iniciativas que les permitan explorar nuevos mercados y con ello crear una nueva ruta en sus modelos organizacionales, la implementación de nuevas tendencias y oportunidades que se gestan a través de la colaboración entre las distintas entidades socias de la agencia y del Sistema Vasco de Innovación.

Como lo plantea la misma Agencia Innobasque, el plan estratégico actual establecido en el País Vasco llamado Plan 2020 tiene como objetivo mejorar el bienestar y crecimiento económico sostenible y el empleo mediante una política de innovación basada en la especialización inteligente y en la mejora de la eficiencia del sistema vasco de Ciencia, Tecnología e Innovación (CTI) (Villate, 2017).

\section{b Evolución del ecosistema del país vasco}

En el año 1980, se creó el primer Gobierno Vasco, tras la aprobación del Estatuto de Autonomía, a partir de esta fecha, cabe distinguir tres grandes períodos (Valdaliso, 2013). 
El primer período, 1980-1990, es la fase en que el nuevo gobierno, además de tener que construir el entramado administrativo regional, debe afrontar la grave crisis que desde la segunda mitad de los 70 del siglo pasado, sacude a la economía vasca y la entrada en la Comunidad Europea en 1986. Debe resaltarse en este período, la decidida apuesta que efectúa el Gobierno Vasco por el mantenimiento de sus sectores industriales tradicionales, dirigidos principalmente en torno al metal: siderurgia, construcción naval, forja, fundición, máquina-herramienta. El Gobierno Vasco impulsó una decidida política de reestructuración que permitió la renovación y el retorno a la viabilidad de buena parte del tejido industrial vasco. Fue una política que no radicó tanto en mejoras de productividad obtenidas por la aplicación de actividades de $\mathrm{I}+\mathrm{D}$, sino a actividades de innovación más tradicional (inversiones en maquinaria y mejoras organizativas, principalmente) (Orkestra, 2018).

El período 1991-98 fue un período de transición, en el que las políticas no fueron tan reactivas como en el período anterior, sino que existió un plan formal de estrategia industrial. En lo que respecta a las políticas de especialización diversificada, el hecho más destacado fue la puesta en marcha de una pionera y decidida política de clusterización. La mayor parte de la misma se orienta a sectores y actividades tradicionales de la economía vasca, que, aunque incluía la definición de proyectos estratégicos de I+D por las asociaciones (clústeres) creadas al respecto, en lo fundamental, la actividad se centró en el impulso a la cooperación y a actuaciones en ámbitos como la mejora de la calidad y la internacionalización.

Por último, el período que va desde 1999 hasta la actualidad ha sido un período en el que prevalece la existencia de planes o estrategias formales de competitividad. Para su diseño se estableció un proceso participativo. Es una estrategia que perseguía llevar a cabo el paso de la economía vasca de un estado competitivo basado en la eficiencia a otro basado en la innovación. Como actuación realmente novedosa, se inicia, explícitamente, una política de diversificación estratégica basada en la $\mathrm{I}+\mathrm{D}$, recogida en los planes de ciencia, tecnología e innovación que se aprueban en el nuevo milenio y que son compartidas por todo el ecosistema.

Desde este análisis, se puede deducir que, en relación al estado de desarrollo en que se encuentre la región, las estrategias de especialización inteligente varían y no descansan principalmente en la $\mathrm{I}+\mathrm{D}$; además del diseño colaborativo.

\section{c. Las estrategias específicas de diversificación basadas en $\mathbf{I}+\mathbf{D}$}

Si bien en el Plan de Ciencia, Tecnología e Innovación (PCTI) 2001-2004 del Gobierno Vasco aparecía una serie de prioridades en materia de ciencia, tecnología e innovación, dentro de las cuales se encontraban algunas de las tecnologías de uso genérico (TIC y biotecnología, en particular), es en el Plan de Ciencia, Tecnología e Innovación 2020 en el que expresamente aparece, como una de sus cuatro grandes apuestas, la de impulsar desde la ciencia y la tecnología nuevos sectores que contribuyan a la diversificación (Gobierno Vasco, 2014).

En el plan se dice que a diferencia de las actuaciones que se enmarcan dentro del objetivo de mejora de la competitividad y que podrían considerarse como "demand pull" dado que su desarrollo está dirigido a dar respuestas a las necesidades y oportunidades de las empresas, las actuaciones desarrolladas en este bloque pueden ser consideradas como "science push" o "technology push”. En este caso, la labor de promoción y liderazgo recae en un primer momento en la propia administración (Gobierno Vasco, 2014).

Otro notable rasgo de la estrategia de diversificación basada en I+D en el País Vasco es que la apuesta en investigación básica ligada a dicha estrategia, en lugar de canalizarla a través de la universidad, tal como propugnaría el modelo de la Triple Hélice (Etzkovitz y Leydesdorff, 2000; Etzkowitz, 2003), se canalizó en gran medida a través de la creación de Centros de Investigación Cooperativa (CIC), cuya investigación básica orientada era financiada mayoritariamente por el Departamento de Industria y no por el de Educación.

El País Vasco apuesta fuertemente por convertirse en una economía del conocimiento y la innovación, abierta a los mercados internacionales, con una base industrial sólida y de elevado contenido tecnológico.

\section{d. Papel de las Universidades (, Mondragón, Deusto y País Vasco)}

Las universidades en el País Vasco son parte importante del ecosistema y cumplen roles complementarios que contribuyen al logro de las metas del País Vasco y de la RIS3. 
Universidad de Mondragón: es una universidad cooperativa sin fines de lucro. Los funcionarios y profesores son los dueños de la universidad y ofrece algunas carreras trilingües: español, euskera e inglés. Se financia un tercio por la matrícula, un tercio por proyectos de transferencia y un tercio por proyectos de cooperación.

El modelo educativo consta de cinco objetivos en la educación del individuo:

1. Multicomunicador: énfasis en la resiliencia y la interculturalidad

2. Ciudadanos del mundo: preparación para los retos mundiales

3. Identidad y objetivo: se forma al individuo dando énfasis en la parte humanista más que en la formación misma.

4. Aprendiz activo: herramientas para aprendizaje continuo a través de la vida.

5. Persona cooperativa: no se le prepara para que se acomode en un sistema sino para que lo transforme.

De los 10 meses de lecciones al año, los tres primeros meses del primer año de carrera se dedican a realizar un proyecto en una empresa, el tiempo se amplía a 6 meses en la empresa en los años siguientes hasta que en el último año de carrera se realiza todo el proceso en la empresa. Para esto, se realizan convenios con las empresas y estas le pagan al estudiante. También, realizan prácticas internacionales en Finlandia, Estados Unidos (específicamente, en Silicon Valley), China, India y otros países. Los profesores son en realidad coaches, donde aplican un modelo educativo innovador y esto ha generado una alta empleabilidad. Realizan investigación aplicada de muy alta calidad; se constituyen en la primera universidad en transferencia en España y la sétima en Europa. Para el año 2017, el $60 \%$ de tesis de doctorados fueron financiados por las empresas, 613 empresas recibieron capacitación en educación continua de la universidad y adicionalmente se realizan múltiples publicaciones en conjunto con las empresas (Axtra y Altuna, 2017).

La universidad cuenta con la Incubadora Iratxe Saiolan que se creó en la Facultad de Ingeniería, para colaborar con nuevas empresas. Se encuentra ubicada en el parque tecnológico Garaia y entre su personal hay profesores de la universidad. Este se encarga de buscar nuevas ideas en el mercado y promover proyectos entre las empresas y la universidad. También, la incubadora cuenta con una antena internacional para atraer ideas del exterior y tienen laboratorios a disposición para la investigación y la realización de prototipos. Para el 2017, se atendieron 101 proyectos en total: 31 fueron presentados por personas emprendedoras y, los otros 70, propuestos por empresas, lo que evidencia la importancia de la vinculación.

Universidad de Deusto: es una universidad privada fundada hace más de 100 años que trabaja bajo el esquema RIS3 con las áreas estratégicas y las áreas no prioritarias con énfasis en proyectos interdisciplinarios que se pueden encadenar unos con otros. Está asociada a la Compañía de Jesús y se inserta así en una red universitaria de alcance mundial y en una tradición pedagógica centrada en la persona como valor preeminente (Universidad de Deusto, 2019). Dentro del horizonte universal de los saberes y del interés general por lo humano, esta Universidad pretende servir como institución universitaria, tanto en sus necesidades culturales, como en el desarrollo social, técnico y económico.

En 2018, obtuvo el puesto mundial número seis en excelencia docente - Global Teaching Excellence Award 2018 (Biskaia Talent, 2019). Dentro de la Universidad se encuentra el Instituto Vasco de Competitividad, Orkestra, el cual ofrece talleres de innovación y emprendimiento para todas las carreras y apoya el esfuerzo mediante metodologías novedosas como el uso de la teoría de juegos y mediante el arte.

Universidad País Vasco: es una universidad pública centrada en la creación de nuevos conocimientos y su transferencia a la sociedad, está en el quinto lugar de creación de empresas en España, con un promedio de creación de siete empresas y una tasa de sobrevivencia en los primero cinco años del $80 \%$. La universidad diseña y acompaña los proyectos con el soporte y la capacitación requeridas como parte de una alianza público-privada para desarrollar nuevos vínculos y captar fondos para la universidad llamado Euskampus (Campillo, 2017). En 2010, la Universidad del País Vasco, junto con Tecnalia Technology Corporation y Donostia International Physics Centre (DIPC), lanzaron el proyecto Euskampus, el cual es un centro de excelencia internacional. Las tres misiones convergen en una visión para establecer una asociación duradera y responsable mediante la conformación de una alianza estratégica que promueva un programa de especialización inteligente real

Euskampus dinamiza el afloramiento de ideas y líneas de trabajo comunes, activa capacidades y apoya los polos de 
conocimiento de forma constante y simultánea. Ejerce una doble función, por una parte, es la entidad dinamizadora de la comunidad, pues se encarga de trabajar con los investigadores de las entidades participantes mediante metodologías de co-creación para establecer los marcos estratégicos y las agendas de actuaciones, realizar el seguimiento, monitoreo y evaluación de los polos y, por otra parte, funciona como un "socio inversor" que gestiona un fondo de inversión. Este recurso se ejecuta como capital semilla para impulsar y acelerar la ejecución de las actuaciones contempladas en los polos, cuyo retorno serán los indicadores de los cuadros de mando de las entidades participantes (Campillo, 2017). Con la incorporación de la Universidad de Burdeos (Francia) se potencia la cooperación internacional y se genera un Campus Transfronterizo Bordeaux-Euskampus.

\section{e Rol de los centros de desarrollo tecnológico}

En la transformación desarrollada en el País Vasco, con el fin de impulsar la I+D+i, se crean los centros tecnológicos ligados a las políticas de tecnología e innovación progresivas y a una red de centros de investigación, centros

Figura 2.

Misión de las instituciones que conforman el proyecto Euskampus

\section{Universidad del País Vasco \\ Tecnalia \\ Donostia International Physics Centre (DIPC)}

Empresa tecnológica comprometida con la industria vasca para abordar la transformación del conocimiento en PIB y mejorar el bienestar empresarial al crear oportunidades de negocio.

Objetivos: Proporcionar a la sociedad en general y a la vasca en particular, una oferta de formación de postgrado reconocida a nivel internacional, sensible a la demanda y que contribuya al incremento de la competitividad, al desarrollo sostenible y a la cohesión social de su entorno mediante: Una gestión eficaz y transparente de los recursos encomendados.

Una amplia oferta formativa de calidad. La formación integral de personas impulsando la excelencia y la interdisciplinariedad.

La difusión de la oferta formativa y la promoción de la empleabilidad y la inserción laboral.

El fomento de relaciones con universidades y organizaciones de investigación y formación de reconocido prestigio internacional.

(Informacihttps://www.ehu.eus/es/we$\mathrm{b} / \mathrm{mde} / \mathrm{xedea} \mathrm{l}$ ) (https://www.tecnalia.com/es/tecnalia/vision-estrategica.htm)
Centro de investigación que se enfoca en promocionar y catalizar el desarrollo al más alto nivel de la investigación básica y básica-orientada en ciencia de materiales. Desde su creación, el DIPC ha sido una institución abierta y ligada a la Universidad del País Vasco, que sive como plataforma de internacionalización de la ciencia básica en el País Vasco en el campo de los materiales.

(https://www.science.eus/es/institutions/dipc-donostia-international-physics-center)

Fuente: elaboración propia. 
tecnológicos y unidades de I+D empresarial. La misión de estas unidades es la generación, captación y transferencia de conocimiento científico-tecnológico, con el fin de ponerlo a disposición de las empresas para que puedan complementar sus capacidades tecnológicas, y así mejorar su competitividad (Erdozain, 2017).

Los centros tecnológicos se crean con el objetivo de desarrollar investigaciones que aporten conocimiento y soluciones tecnológicas, se encuentran situados en dos de las tres provincias del País Vasco, en la provincia de Guipúzcoa se encuentran seis de los once y en la provincia de Vizcaya se encuentran los restantes cinco. Un ejemplo de estos centros es el IK4 Research Alliance (Gaiker), constituido por una red de nueve centros tecnológicos en País Vasco que desarrollan $\mathrm{I}+\mathrm{D}+\mathrm{I}$, capturan recursos y personas para cualificarlas. El IK4 ayuda a los centros a cumplir su misión, estos laboratorios están cerca de las zonas industriales y tiene la función de desarrollar tecnología propia que sirva para las empresas, así como formar investigadores. Las empresas contratan personas que se han capacitado en IK4.

Otro ejemplo es la Corporación Tecnalia, que cuenta con un sistema de innovación, el cual incluye agentes para desarrollar diferentes actividades con las universidades, empresas, clústeres, centros de investigación cooperativa, centros científicos y tecnológicos, Innobasque y unidades de I+D empresariales.

\section{f. Rol de los Parques Tecnológicos}

Para alcanzar el objetivo común del País Vasco no solo se requiere contar con una decisión consensuada entre los diferentes actores proponentes de la innovación, sino que también es perentorio disponer de infraestructura adecuada para propiciar un asentamiento idóneo para todos aquellos emprendedores que con sus novedosos proyectos potencian la innovación. En Euskadi, ese requerimiento se solventó a través de la atracción de inversión local y extranjera para desarrollar parques tecnológicos con el fin de coadyuvar con el desarrollo institucional vasco y estimular el emprendimiento. Hay tres parques tecnológicos que el País Vasco logró construir en cada una de sus provincias, a saber: Álava, Guipúzcoa y Vizcaya y se denominan Parke Araba, Parke Gipuzkoa y Parke Bizkaia, respectivamente. Estos son parte de la infraestructura implementada en la región vasca que obedece a un plan estratégico para desarrollar una red de parques tecnológicos que vinieran a dar albergue a todas aquellas pequeñas, medianas y grandes empresas; locales y multinacionales, todas con una orientación a la investigación, al desarrollo y a la innovación.

Actualmente, los tres parques tecnológicos albergan a 429 empresas y generan alrededor de 16.000 empleos directos (Erdozain, 2017). Los parques se reúnen una vez por año para dar a conocer sus proyectos y realizan videoconferencias durante el año para valorar algunos proyectos conjuntos, participan en varias redes nacionales e internacionales y ayudan a internacionalizar a las empresas, por medio cross mentory entre parques.

En definitiva, se ha demostrado los beneficios que puede llegar a tener una región a través del desarrollo de parques tecnológicos y de la implementación de la variable "innovación" como un estandarte idóneo para incubar nuevos emprendimientos generadores de empleo, cultura, nuevas tecnologías, desarrollo económico-social, infraestructura, entre otros, todo en aras de remozar regiones en pro de la sociedad. A la vez, son muchos los esfuerzos que deben realizarse por parte de las comunidades interesadas para integrar a muchos agentes o actores como la industria, comercio, academia, gobiernos locales y gobierno central e incluso organizaciones internacionales, que en conjunto y mediante esfuerzos combinados puedan lograr la consecución de un objetivo común consensuado y orientado, para dirigir de forma sostenida el desarrollo de nuevas empresas tecnológicamente avanzadas, que brinden a su vez nuevas y mejores oportunidades a las futuras generaciones de profesionales en muchos y los más diversos campos de la innovación. Para ello, los parques tecnológicos colaboran con las distintas instituciones del país promoviendo los intereses o vocaciones científicas en los colegios y escuelas a través de módulos interactivos y talleres en áreas como la robótica y energías renovables entre otras. Cabe mencionar que en cada uno de los tres parques tecnológicos, hay varias incubadoras de empresas que promueven el espíritu emprendedor en la región. Además, se gestiona el "mentoring" para compartir el talento de los expertos de las distintas empresas en temáticas de actualidad, promover el liderazgo en la resolución de conflictos y además promover la transferencia de conocimiento entre las empresas. El objetivo final en este sentido es que el "cross mentoring" entre parques tecnológicos permita generar más negocios y encadenamientos productivos. 


\section{CONCLUSIONES}

Puede desprenderse de los resultados evidenciados que el País Vasco apuesta fuertemente por convertirse en una economía del conocimiento e innovación, abierta a los mercados internacionales, con una base industrial sólida y de elevado contenido tecnológico, lo que es clave en el desarrollo económico, social y cultural de un país para obtener altos niveles de crecimiento y bienestar.

Parte de lo aprendido en esta región innovadora es considerar la gestión realizada por tantos actores comprometidos para convertir al País Vasco en un modelo de innovación exportable a todo el continente europeo y a otras latitudes, es que necesariamente, debe integrarse en una sola iniciativa y tomar la decisión de implementar una idea clara, precisa y bien orientada, sustentada, equitativamente, por todos y cada uno de los actores civiles y públicos. Además, se debe alimentar de una estrategia bien definida de lo que se quiere desarrollar por medio de la innovación y, a su vez, deslindarse del interés de beneficiar a unos pocos e impactar al interés público, por medio de todas aquellas ventajas y oportunidades que puedan identificarse como puntos fuertes y que figuran como activos de la comunidad. Es decir, se requiere socializar la innovación en función del bienestar público y privado como producto o rentabilidad de esa alianza estratégica entre actores de naturaleza disímil.

Para crear un modelo de innovación como el que ha logrado el país Vasco, impulsado por Innobasque y los otros agentes, se requiere de muchos factores, dentro de los cuales podemos citar: inversión, encadenamientos productivos entre demandantes y oferentes de bienes y servicios, fomento de la ciencia y tecnología, infraestructura, áreas de oportunidad vinculadas con los territorios (la industria agroalimentaria, ecosistemas, hábitat urbano), grupos mixtos de trabajo, investigación y desarrollo, academia, gobierno local, actores civiles, entre otros.

Del análisis del caso del País Vasco se desprende, por una parte, que en un único territorio pueden coexistir diferentes modalidades de estrategias de diversificación basadas en la $\mathrm{I}+\mathrm{D}$, y que en cada una de ellas el papel del gobierno y de los restantes agentes diferirá en función de las capacidades empresariales y científico-tecnológicas disponibles en la región. Por otra parte, cuando existen esas capacidades en el sector privado o universitario, el gobierno puede adoptar un papel principalmente de facilitador, en otros casos, en que tales capacidades eran cuasi inexistentes o puramente potenciales, el papel del gobierno es mucho más activo y provocador.

Otro de los factores del éxito de las estrategias seguidas por el País Vasco, que hacen tal iniciativa difícil de imitar por otras regiones, radica en el elevado grado de competencias de su gobierno regional, que le permiten actuar de modo coordinado en múltiples de los factores clave de éxito para las iniciativas. Además, el País Vasco ha disfrutado, en particular, de una capacidad de inversión muy grande y sostenida a lo largo del tiempo, posibilitada por su peculiar régimen de concierto económico y el progreso que muestra su autonomía como región.

El papel de las universidades es clave en el ecosistema del País Vasco ya que todas tienen objetivos complementarios entre sí: la de Mondragón se especializa en el sector industrial, la de Deusto en capital social y la País Vasco en ciencia y tecnología. Estas instituciones cumplen un rol esencial en la agenda de desarrollo en el País Vasco donde no se replica sin más el modelo de las regiones exitosas en sus ámbitos, sino que la estrategia se ha adaptado a las circunstancias de cada región.

De lo anterior, se infiere que para alcanzar un objetivo común que venga a beneficiar a los actores del ecosistema, se requiere de forma indubitable de la participación sincera y del compromiso inquebrantable de todas las partes involucradas, ya que el sistema no funciona como un archipiélago, sino como un solo territorio con un objetivo común planteado por muchos y para muchos, el cual mezcla ese cúmulo de elementos que serán la génesis de un nuevo territorio liderado por la innovación y el interés común de aquella pluralidad vasca en pro de la calidad de vida de sus habitantes.

En el caso de Costa Rica, un país pequeño conformado geográficamente por siete provincias, donde cada una cuenta con sus fortalezas, debilidades, recursos e infraestructura, también cuenta con un plan de desarrollo regional en donde interactúan gobiernos locales (municipalidades), empresas, sector socio productivo, instituciones públicas, bases sociales y universidades. Además, algunas regiones están más desarrolladas que otras; por tanto, hace falta definir políticas claras a nivel nacional para valorar las potencialidades de cada zona y realizar un inventario de 
los recursos con los que cuenta, la infraestructura que tiene y qué haría falta para potenciar el desarrollo de una estrategia inteligente y atraer nuevas inversiones.

En las diferentes regiones se cuenta con iniciativas o con plataformas implementadas de desarrollo regional, esto es un inicio para articular y trabajar con todos los sectores para priorizar de acuerdo a las potencialidades y fortalezas de cada región y para tomar en cuenta la innovación, investigación, conectividad, desarrollo tecnológico y liderazgo colaborativo. De acuerdo con la realidad de Costa Rica y la experiencia exitosa del País Vasco, es importante valorar qué estrategia puede adaptarse basada en este modelo de especialización inteligente, con el fin de aprovechar al máximo los recursos y así mejorar la calidad de vida de los costarricenses. Costa Rica cuenta con las zonas económicas especiales en diferentes regiones del país. Estas iniciativas involucran tanto al gobierno como al sector socio productivo y, en algunos, casos a la academia y a la sociedad civil, juntos se articulan con objetivos comunes de desarrollo y buscan generar empleos de calidad en la región, por lo que la estrategia permite implementar mejores condiciones para la inversión extranjera. En resumen, estas son iniciativas que requieren de mayor apoyo y planificación para que pueda utilizar el modelo de especialización inteligente

\section{REFERENCIAS}

Axtra, V., Altuna, J. Entrevista personal el 21 de noviembre 2017, Universidad de Mondragón.

Biskaia Talent. La Universidad de Deusto en el top 6 mundial de la excelencia docente. Recuperado el 11 de setiembre de 2019 de https://www.bizkaiatalent.eus/deusto-global-teaching-award/

Campillo, I. Entrevista personal el 23 de noviembre 2017. Euskampus - UPV/EHU.

Campos, Y. (2017). Taller "Especialización Inteligente: Costa Rica hacia polo de innovación”. 3 de noviembre del 2017. Universidad Estatal a Distancia.

Euskadi (2014). Prioridades estratégicas de especialización inteligente (RIS3), Gobierno Vasco, España.

Etzkowitz, H. y Leydesdorff, L. (2000). The dynamics of innovation: from National Systems and "Mode 2" to a triple helix of university - industry - government relations. Research Policy 29, pp. 109-123.

Etzkowitz, H. (2003). Innovation in innovation: The Triple Helix of university-industry-government relations. Social Science Information, 42(3), pp. 293-337.

Erdozain, J. M. Entrevista personal 23 de noviembre 2017. IK4 Research Allliance.

Gobierno Vasco (2014). Una Estrategia de Especialización Inteligente 2020, https://www.irekia.euskadi.eus/uploads/ attachments/5585/PCTI_Euskadi_2020.pdf

Instituto Tecnológico de Costa Rica. 2018. Pensis XI Edición. Poderosos instrumentos de desarrollo. https://www.tec.ac.cr/ pensis/articulos/poderosos-instrumentos-desarrollo

Navarro Arancegui, M., Aranguren Querejeta, M. J., Magro Montero, E. (2011). Estrategias de especialización inteligente: el caso del País Vasco. Orkestra-Basque Institute of Competitiveness and Deusto Business School, University of Deusto.

Orkestra (2018). Informe de competitividad del País Vasco. Instituto Vasco de Competitividad.

Universidad de Deusto. 2019. Recuperado de phttps://www.deusto.es/cs/Satellite/deusto/es/universidad-deusto/sobredeusto-0/la-institucion/mision-e-identidad

Urkullu Renteria, I. (2014). Una estrategia de especialización inteligente. Gobierno Vasco. https://www.irekia.euskadi.eus/ uploads/attachments/5585/PCTI_Euskadi_2020.pdf

Valdaliso Gago, Jesús M. (2013). El “caso vasco” de Harvard y las estrategias de competitividad del País Vasco desde 1980 hasta 2012. Revista internacional de los estudios vascos, 58 (1), pp. 112-130.

Villate, J. M. Entrevista personal 20 de noviembre 2017. Funciones de la Agencia Innobasque en el País Vasco. 